\title{
Structural and genomic variation in preterm birth
}

Alper Uzun, Yavuz Sahin, Jessica S. Schuster, Xiaojing Zheng, Kelli Ryckman, Eleanor Feingold and James Padbury

Pediatric Research advance online publication, August 24, 2016; doi:10.1038/pr.2016.152; accepted article preview online July 24, 2016

In the published version of this manuscript, author Xiaojing Zheng's affiliation was incorrect. The correct affiliation is Department of Pediatrics, School of Medicine, University of North Carolina at Chapel Hill, Chapel Hill, North Carolina. The publisher regrets the error. 